Rev Biomed 2004; 15:69-72.

\title{
Bajo índice de publicaciones en \\ las escuelas de medicina de Venezuela: el caso del Núcleo Bolívar de la Universidad de Oriente.
}

\author{
Carta al Editor
}

\author{
Rodolfo A. Devera
}

Depto. de Parasitología y Microbiología, Escuela de Medicina, Universidad de Oriente, Núcleo Bolívar, Ciudad Bolívar, Venezuela.

Muchos profesores e investigadores encargados de los Consejos de Investigación o similares, de las diversas Universidades de Venezuela suelen preguntarse por qué en las Escuelas o Facultades de Medicina se publica tan poco. Las razones son múltiples y para discutirlas consideraremos el ejemplo de la Escuela de Medicina de la Universidad de Oriente (EM-UDO), núcleo de Bolívar, ubicada en Ciudad Bolívar, capital del estado Bolívar. Esta Escuela fue fundada el 8 de enero de 1962, cuatro años después de la creación de la Universidad de Oriente (1). Desde entonces esta escuela ha manteniendo un elevado prestigio con relación a la calidad de sus egresados.

A primera vista la respuesta de por qué no se publica pudiera ser simple: porque no se hace investigación y, por tanto, no hay nada que presentar (publicar) a la comunidad científica. Sin embargo, esta no es la razón. Veamos por qué: entre 1997 y 2001 se han graduado en promedio 60 médicos cirujanos (pre-grado) por año y por normas internas de la institución para obtener el grado de médico cada bachiller debe realizar una tesis de grado, la cual no es más que un trabajo de investigación que requiere de la asesoría de un docente-investigador de la Universidad. Esto indica que entre 1997 y 2001 se elaboraron aproximadamente 350 tesis de grado en la carrera de Medicina. Este trabajo en su fase de proyecto es evaluado por una comisión que determina el rigor científico, la pertinencia, viabilidad y calidad del trabajo a desarrollar. Finalmente, para ser aprobada, la tesis pasa por la evaluación de tres profesores de la institución que constituyen el jurado de ese trabajo.

Igual sucede a nivel de postgrado, en todos los cursos de especialización y de maestría impartidos en la Escuela de Medicina. Anualmente se forman decenas de especialistas, cada uno de los cuales debe presentar un trabajo científico para poder obtener su diploma.

$\mathrm{Si}$ a lo anterior agregamos que para ascender en el escalafón docente, todo miembro del personal de la EM-UDO debe presentar un trabajo de ascenso,

Solicitud de sobretiros: Rodolfo Devera. Departamento de Parasitología y Microbiología, Escuela de Medicina, Universidad de Oriente, Núcleo Bolívar. Av. José Méndez. Ciudad Bolívar, Estado Bolívar, Código Postal: 8001-A. Venezuela.

E-mail: RodolfoDevera@hotmail.com

Recibido el 14/Marzo/2003. Aceptado para publicación el 16/Diciembre/2003.

Este artículo está disponible en http://www.uady.mx/sitios/biomedic/revbiomed/pdf/rb041519.pdf

Vol. 15/No.1/Enero-Marzo, 2004 


\section{RA Devera.}

el cual es sometido a evaluación externa (profesores de otras universidades), se concluye que los profesores también deben hacer investigación (2).

Por lo tanto, sí se realizan trabajos de investigación de elevada calidad en nuestra escuela de Medicina. El problema está en que ese trabajo no se publica y por tanto, no se da a conocer, y en ciencia lo que no se publica no tiene valor $(3,4)$. Así, un sinnúmero de trabajos de gran calidad descansan en los archivos de profesores o en estantes polvorientos de nuestra biblioteca o de las salas de los diferentes jefes de Departamento, sin que el resto de los investigadores tengan acceso a ese conocimiento.

Veamos algunos indicadores del bajo porcentaje de publicaciones por investigadores de la EM-UDO.

\section{Artículos publicados en Revistas} Indexadas (MedLine). Se realizó una revisión bibliográfica en el MedLine de los años 1997 a 2001 utilizando las palabras claves: Universidad de Oriente, Bolívar y UDO. Los resultados mostraron 21 trabajos realizados por docentes investigadores de la Escuela de Medicina durante estos 5 años, con un promedio de 4,2 trabajos publicados por año. Estos trabajos se distribuyeron apenas en 4 departamentos, recordando que la Escuela de Medicina cuenta con 14 departamentos. Once artículos $(53,38 \%)$ fueron publicados por docentes del Depto. de Parasitología y Microbiología, $5(23,81 \%)$ por el Centro de Microscopía, 3 (14,28\%) por el Depto. de Ciencias Fisiológicas y $2(9,52 \%)$ por el Depto. de Medicina. Considerando el número de profesores por cada uno de estos departamentos, se verifica la relación de trabajos publicados por profesor durante el período comprendido entre 1997 y 2001 (cuadro 1). La mejor relación con 1,25 trabajos durante ese período por profesor se obtuvo en el Centro de Microscopía Electrónica que publicó 5 trabajos y cuenta con 4 docentes. Después le sigue el Depto. de Parasitología y Microbiología que con 9 docentes publicó 1,22 trabajo durante ese período. Resalta el Depto. de Medicina con el mayor número de profesores (29) de todos los departamentos de la Escuela con apenas un promedio de 0,07 trabajos publicados por profesor. Considerando los 14 departamentos y los 140 docentes de la EM-UDO la relación de trabajos publicados por profesor fue de apenas 0,15 trabajo por profesor.

\section{Trabajos publicados en la Revista}

SABER. Otro índice a considerar se refiere a los trabajos publicados en la Revista SABER que es el órgano científico de divulgación del Consejo de Investigación de la Universidad de Oriente. Es una revista multidisciplinaria arbitrada que publica trabajos de diferentes áreas. Entre 1997 y 2001 apenas 6 trabajos elaborados por docentes de la EM-UDO fueron publicados en la revista, con una media en ese período de 1,2 trabajos. Entonces nos preguntamos: ¿dónde están todas esas tesis y todos esos trabajos de ascenso?

\section{Programa de Promoción al Investigador} (PPI). A lo anterior tenemos que agregar que en la ultima convocatoria del Programa de Promoción al Investigador (PPI), de la Fundación Venezolana de Promoción del Investigador del Ministerio de Ciencia y Tecnología, en el año 2000, apenas fueron reconocidos 11 profesores de la EM-UDO.

Entonces, se hace investigación pero no se publica. Para tratar de explicar las causas del porqué nuestros profesores no publican tendríamos que considerar varias razones:

\section{Falta de capacidad y temor a la evaluación} de sus pares. Muchos colegas sienten aprensión y hasta temor ante el posible rechazo de su trabajo durante el proceso de arbitraje. Otros creen que su trabajo es de poco valor y no podría ser publicado (3). Aunque en verdad hay docentes poco capacitados para escribir o que temen las críticas, consideramos que ambas situaciones son erróneas, pues ¿de qué otra forma se mide la calidad de nuestro trabajo si no es mediante la evaluación por nuestros pares? Este es el mejor control de calidad que se puede tener. Además, cuando el trabajo se realiza con una metodología y un rigor científico adecuados se tienen que producir investigaciones de calidad.

2. Falta de tiempo. Otros sostienen que publicar implica una inversión de mucho tiempo con relación a la preparación y adaptación del manuscrito a una determinada revista. Además, muchos consideran que

\section{Revista Biomédica}




\section{Publicaciones en las escuelas de Medicina de Venezuela.}

Cuadro 1

Artículos publicados en revistas indexadas en el MedLine, realizados por docentes de la EM-UDO según Departamento. Período 1997 a 2001.

\begin{tabular}{lccc}
\hline Departamento & $\begin{array}{c}\text { Artículos } \\
\text { Publicados* }\end{array}$ & No. profesores & $\begin{array}{c}\text { Relación } \\
\text { Artículo/Profesor }\end{array}$ \\
\hline Centro de Microscopía Electrónica & 5 & 4 & 1,25 \\
Parasitología y Microbiología & 11 & 9 & 1,22 \\
Ciencias Fisiológicas & 3 & 21 & 0,14 \\
Medicina & 2 & 29 & 0,07 \\
Otros 10 Dptos. & 0 & 77 & 0 \\
Total & 21 & 140 & 0,15 \\
\hline
\end{tabular}

* Solo se utilizó el primer autor. Si el trabajo fue realizado en varios departamentos se consideró el departamento del autor principal.

Total de Profesores en la carrera de Medicina, EM-UDO.

* Aunque este centro está adscrito al Dpto. de Medicina, funciona en realidad como un departamento independiente en la EM-UDO.

están sobrecargados de actividades docentes y/o asistenciales. Todas son simples excusas, cuando se quiere podemos organizarnos para cumplir las metas planteadas. Además, dentro de nuestra universidad existe la posibilidad de descargar horas docentes por concepto de investigación.

3. Escasez de recursos económicos. Dependiendo de la naturaleza de la investigación ésta puede ser costosa y difícil de realizar si no se cuenta con el financiamiento respectivo. Aunque existen varios entes financiadores nacionales e internacionales, ante el temor y las críticas muchos docentes de la EMUDO se limitan a solicitar financiamiento de forma local ante sus universidades. Siendo que en realidad un porcentaje ínfimo del Producto Interno Bruto (PIB) venezolano es destinado para investigación (5). Por otro lado, elaborar un manuscrito de calidad para ser sometido a consideración en una revista de prestigio requiere dinero. Desde la traducción del artículo hasta la impresión, preparación de fotos, figuras, etc. Todos estos gastos son muchas veces difíciles de ser sufragados por el docente.

4. Falta de apoyo institucional. Sabemos del esfuerzo, entre las limitaciones existentes, que el Consejo de Investigación de la Universidad de Oriente ha realizado para intentar incrementar el número de Investigadores Activos (IA) y de investigadores acreditados en el Sistema de Promoción al
Investigador (PPI) (6). Pero, tal vez este Consejo no esté prestando todo el apoyo necesario al investigador. Para citar un ejemplo, considérese el siguiente caso: cuando se somete un proyecto ante el Consejo pasan varios meses (hasta años) para saber si fue o no aprobado. Puede ser aprobado pero no se tiene el dinero, o con el monto asignado ya no es posible comprar lo presupuestado. Aunado a esto hay que agregar la inmensa maquinaría burocrática que hay que salvar para obtener los recursos y realizar las compras de equipos y materiales (5). Además de la falta de apoyo en la fase de elaboración de los manuscritos, ya comentado anteriormente.

5. Generación de relevo inexistente. A lo largo de su historia la EM-UDO ha contado con investigadores que aunque pocos, han tenido su labor reconocida y ganada mediante sus méritos. Lamentablemente, estas mismas personas no han dejado generación de relevo a quienes leguen parte de sus conocimientos así como sus líneas de investigación. Las instituciones también han tenido una cuota de responsabilidad ante la ausencia de políticas adecuadas para formar personal docente de relevo.

6. Poca motivación. Creemos, que junto con las razones de índole económica, ésta sea la razón principal que justifica el poco volumen de publicaciones en nuestra escuela. Existe una ausencia de motivación por parte del docente, de la institución y de las 


\section{RA Devera.}

autoridades. Hay innumerables obstáculos para poder obtener subvención de un proyecto de investigación, no se cuenta con el personal técnico que apoye las investigaciones, el trabajo es poco valorado y reconocido tanto dentro como fuera de la institución. Además de los ya citados obstáculos el verdadero docente investigador debe lidiar con colegas improductivos, que son la mayoría, y para quienes no se aplica el dogma: el que escribe, sobrevive, ya que han vivido de la Universidad sin realizar labores de investigación, siendo simples repetidores de conocimientos generados por otros $(3,4)$. En Venezuela, todo docente universitario debe investigar, sin embargo, vemos como una buena parte de los profesores nunca ha realizado un trabajo de investigación. Además de la inexistencia de sanciones, los salarios son iguales para ambos profesionales, por lo tanto se motiva la no investigación.

Con relación al reconocimiento, se debe valorar el esfuerzo que la institución(Universidad de Oriente) viene realizando en los últimos años al intentar reconocer la labor docente mediante incentivos económicos (Premio de Estimulo al Investigador-PEI). Sin embargo, además de insuficientes, con el pasar del tiempo los baremos de estos premios se han tornado más rigurosos habiendo un contraste entre el nivel de exigencia y la realidad de la Universidad.

Como se aprecia, es un conjunto de factores y razones que explican el reducido número de publicaciones por parte de los docentes de la EMUDO. Si bien son razones valederas deben ser combatidas con medidas enérgicas que no sólo deberían emanar del Consejo de Investigación, promoviendo, facilitando y reconociendo aun más la labor investigativa en la Universidad. Sino que se requiere de cambios profundos en el seno de la Universidad que lleven a que el docente-investigador viva de la Universidad y no para la Universidad (7). Excluyendo las medidas económicas y administrativas y centrándonos apenas en el docente-investigador, algunas de esas medidas podrían ser: exigencia del título de Maestría o Doctorado para ingresar como docente, mantener un número mínimo de publicaciones anuales por parte de esos profesores, poseer un proyecto de investigación aprobado por entes financiadores, contar con un plan de capacitación docente, etc. (7).

Otro problema actual de nuestras universidades, es que las carreras docentes y de investigación son poco atractivas económicamente hablando para los profesionales que se forman anualmente y que podrían ingresar a estas instituciones.

Estas consideraciones, aunque se pueden verificar en los profesores e investigadores de la EMUDO, también pudieran ser válidas para profesores de otros núcleos y de otras universidades de Venezuela y Latinoamérica. Cada uno de nosotros debe hacer un esfuerzo por producir, o sea, investigar y publicar, pues al final, es en las universidades donde se genera conocimiento para que un país pueda desarrollarse.

Palabras clave: Bibliometría, publicaciones médicas, Venezuela.

\section{REFERENCIAS.}

1.- Ponce Ducharne LD. Escuela de Medicina de la Universidad de Oriente. Apuntes para su Historia. Cumaná: Editorial Universitaria; 1987. p. 61.

2.- Gaceta de la Universidad de Oriente. Reglamento del Personal Docente y de Investigación. 1992; No. 70 extraordinario. p. 46.

3.- Romero R. El Profesor Universitario: ¿Docente o Investigador? Invest Clin 1994; 35: 1-2.

4.- Lifshitz A. El que escribe, sobrevive. Rev Med IMSS Mex 1998; 36: 173-5.

5.- Pérez JE. La investigación científica en Latinoamérica. Interciencia 1998; 23: 316.

6.- Anónimo. La Revista SABER (editorial). SABER 2001; 13: 1 .

7.- Eblen-Zajjur A. La Universidad Venezolana: ¿Docencia contra Producción Científica? Acta Cient Venezol 2001; 52: $1-2$.

\section{Revista Biomédica}

